\title{
Valorización de ceniza de bagazo de caña de la industria azucarera Nicaragüense como sustituto parcial al cemento Portland
}

\author{
B.J. Tórrez-Rivas ${ }^{1}$, J.R. Gaitán-Arévaloㅇ, L.J. Espinoza-Pérez ${ }^{1, *}$, J.I. Escalante-García ${ }^{2}$ \\ ${ }^{1}$ Facultad de Ingeniería Química, Universidad Nacional de Ingeniería (UNI) \\ Avenida Universitaria, PO Box 5595, Managua, Nicaragua \\ ${ }^{2}$ Centro de Investigación y de Estudios Avanzados (CINVESTAV), Unidad Saltillo \\ Av. Industria Metalurgica \# 1062, Ramos Arizpe, Coah., C.P. 25900, México
}

(recibido/received: 01-Jul-2014; aceptado/accepted: 25-Aug-2014)

\begin{abstract}
RESUMEN
En este estudio, se presenta la valoración del uso de las cenizas de bagazo de caña (CBCA) proveniente del Ingenio Monte Rosa (Nicaragua) como material alternativo al cemento Portland (CP) mediante estudios de caracterización avanzada y propiedades mecánicas, en pastas endurecidas de CP sustituidas en 15 y $45 \%$ por CBCA; además se utilizó pastas de referencia con $100 \%$ $\mathrm{CP}$ sobre las cuales se evaluó las resistencias mecánicas (RM), el consumo de hidróxido de calcio y el desarrollo de los productos de reacción. Los resultados revelaron que los valores más altos de RM reportados fueron por el sistema de referencia, indicando que la RM disminuyó con el incremento en el porcentaje de sustitución. Por medio de los análisis de difracción de rayos $\mathrm{X}(\mathrm{DRX})$ y análisis térmicos por ATG/ATD fue demostrado el consumo de $\mathrm{Ca}(\mathrm{OH})_{2}$ que dio lugar a la formación de gel CSH como principal producto de hidratación y responsable de la ganancia de las propiedades mecánicas. Los productos de reacción fueron observados también por microscopia electrónica de barrido (MEB) donde el mecanismo en estado sólido fue notable. A partir de estos resultados se puede concluir que las CBCA son un material puzolánico con una mejor reactividad frente al $\mathrm{Ca}(\mathrm{OH})_{2}$ con $15 \%$ de sustitución.
\end{abstract}

Palabras clave: Ceniza de bagazo de caña; Cemento Pórtland; Puzolana

\begin{abstract}
In this study, the use of the ashes of sugarcane bagasse (ASB) from Monte Rosa sugarcane mill (Nicaragua) as an alternative material to Portland Cement (PC) was evaluated. The evaluation was performed through studies of advanced characterization and mechanical properties in pastes with $15 \%$ and $45 \%$ substitution of PC. Moreover, $100 \%$ PC pastes were used as reference. The mechanical resistance (MR), the consumption of calcium hydroxide and the development of reaction products were measured. The results showed that the highest values of MR were obtained for the reference pastes, thus indicating that the MR decreased with increase in the degree of substitution. Through the analysis of X-ray diffraction (XRD) and thermal analysis TGA / DTA was found that the consumption of $\mathrm{Ca}(\mathrm{OH})_{2}$ gave rise to the formation of $\mathrm{CSH}$ gel as the main product of hydration and caused the gain in the mechanical properties. The reaction products were observed by scanning electron microscopy (SEM) where the mechanism in solid state was noticeable. From this result, it can be concluded that the ASB are pozzolanic material with a better reactivity to $\mathrm{Ca}(\mathrm{OH})_{2}$ with $15 \%$ of substitution.
\end{abstract}

Keywords: Sugarcane bagasse; Portland cement; Pozzolan

\footnotetext{
*Autor para la correspondencia. Tel.: +505 22781463.

Correo electrónico: lester.espinoza@fiq.uni.edu.ni (L. Pérez).
} 


\section{Introducción}

El importante crecimiento en la demanda de cemento Portland (CP) en el sector construcción, sobre todo impulsado por la construcción de viviendas de interés social, ha generado un apremiante interés concerniente al aprovechamiento de materiales alternativos que permitan obtener cementos de igual o mejor calidad, logrando disminuir el alto requerimiento energético y la fuerte emisión de contaminantes $(\sim 4,000 \mathrm{~kJ} / \mathrm{kg}$ cemento producido, lo que genera $\sim 0.85-1 \mathrm{~kg}$ de $\mathrm{CO}_{2}$ emitido a la atmosfera por cada $1 \mathrm{~kg}$ cemento producido) por descarbonatación de materia prima y uso de combustibles (Escalante, 2002).

Ciertos autores han encontrado que son muchos los beneficios que la adición de puzolana le confiere al cemento: mejoramiento de la trabajabilidad, menor calor de hidratación (Carmona, 2011), mayor resistencia a los sulfatos, mejores resistencias mecánicas (Alujas et al., 2010), costos de producción más bajos lo que significa menores precios para el consumidor, requerimiento de menos tecnología y equipos ya que en el caso de las cenizas de bagazo de caña (CBCA) para su implementación se requiere ya sea de molienda o tamizado (Payá, 2012).

Estudios realizados con diferentes residuos agrícolas de la industria azucarera tales como: cenizas de cascarilla de arroz, cenizas de paja de caña y cenizas de bagazo de caña (Martinera et al., 1998) demuestran su potencial uso por las propiedades puzolánicas que poseen originadas debido a la calcinación de las mismas, formándose así compuestos de óxidos ácidos, principalmente de dióxido de silicio (Payá, 2012) y que de acuerdo a su definición una vez divididas finamente $\mathrm{y}$ en presencia de agua reaccionan químicamente con el hidróxido de calcio que aporta la hidratación del CP una vez adicionadas, para formar compuestos con propiedades cementantes (Quintana, 2005) siendo generalmente un silicato de calcio hidratado (C-S-H) de estequiometria no definida. Sin embargo, la reactividad puzolánica depende en gran medida del área superficial específica, tamaño de partícula y características mineralógicas que a la vez dependen de las condiciones de combustión, de tiempo y temperatura (Morales et al., 2009).

Actualmente en los ingenios azucareros Nicaragüenses existe una gran cantidad de bagazo que es utilizado como combustible para la producción de vapor de alta presión en las calderas, para la producción de energía eléctrica, de lo que resulta otro residuo: las cenizas, que en muchos de los casos no se les da ningún uso o se usan como abono. Datos recopilados por Perdomo (2009) manifiestan que la disposición de cenizas sin humedad se aproxima a los 44719.66 toneladas, dato que en la actualidad seria mayor dado la demanda creciente de azúcar, estas cenizas para su manejo requieren ocupar un área de 1.7 hectáreas, una cantidad de terreno que podría ser útil para otras actividades.

Con base en los argumentos mencionados anteriormente, se trabajó con las cenizas de bagazo de caña de azúcar provenientes del Ingenio Monte Rosa, ubicado en la ciudad de Chinandega, Nicaragua con el fin de evaluar su potencial uso como reemplazo del cemento Portland.

\section{Material y Métodos}

\subsection{Materiales utilizados}

Se utilizó cemento Portland (CP) con una gravedad específica de $3.08 \mathrm{~kg} / \mathrm{m}^{3}$, un área superficial de $3296 \mathrm{~cm}^{2} / \mathrm{g}$ y un tamaño promedio de partícula de $13.84 \mu \mathrm{m}$. La ceniza de bagazo de caña (CBCA) fue obtenida del Ingenio Monte Rosa ubicado en Chinandega, Nicaragua. Las CBCA utilizadas tiene una gravedad específica de $2.13 \mathrm{~kg} / \mathrm{m}^{3}$, un área superficial de $2552 \mathrm{~cm}^{2} / \mathrm{g}$ y un tamaño promedio de partícula de $36.32 \mu \mathrm{m}$, la cual fue determinada por medio de difracción láser. La composición química de las CBCA y del CP fue determinada por fluorescencia de rayos $\mathrm{X}$ y se muestra en la Tabla 1 .

En la Fig. 1 se presenta el patrón de difracción de las CBCA obtenido por difracción de rayos $\mathrm{X}$, se hizo evidente una reflexión muy definida alrededor de $\operatorname{los} 30^{\circ} 2 \theta$ de la fase cristalina albita y reflexiones con menor intensidad 
correspondiente a la fase cristalina cristobalita, que se produjeron como resultado de las altas temperaturas de calcinación por encima de los $800^{\circ} \mathrm{C}$ (Cordeiro et al., 2009), el halo localizado entre los $15^{\circ}$ a $38^{\circ} 2 \theta$ en el difractograma, indicó la presencia de material vítreo en las cenizas en estudio; otros autores han localizado este halo entre los 15 a $30^{\circ} 2 \theta$ (Morales et al., 2009; Cordeiro et al., 2009).

Tabla 1 Composición química de las CBCA y $\mathrm{CP}$ (\% peso).

\begin{tabular}{ccc}
\hline Óxido & CBCA & CP \\
\hline $\mathrm{ZrO}_{2}$ & 0.006 & 0.009 \\
$\mathrm{Cl}$ & 0.017 & 0.037 \\
$\mathrm{SrO}$ & 0.031 & 0.076 \\
$\mathrm{MnO}$ & 0.095 & 0.111 \\
$\mathrm{TiO}_{2}$ & 0.395 & 0.212 \\
$\mathrm{SO}_{3}$ & 0.399 & 4.187 \\
$\mathrm{Na}_{2} \mathrm{O}$ & 0.509 & 0.440 \\
$\mathrm{P}_{2} \mathrm{O}_{5}$ & 1.15 & 0.087 \\
$\mathrm{MgO}$ & 1.59 & 1.04 \\
$\mathrm{CaO}$ & 3.556 & 61.49 \\
$\mathrm{~K}_{2} \mathrm{O}$ & 4.145 & 0.75 \\
$\mathrm{Fe}_{2} \mathrm{O}_{3}$ & 4.921 & 2.343 \\
$\mathrm{Al}_{2} \mathrm{O}_{3}$ & 6.726 & 3.75 \\
$\mathrm{PPI}$ & 14.13 & 11.45 \\
$\mathrm{SiO}_{2}$ & 62.33 & 13.9 \\
$\mathrm{Cr}_{2} \mathrm{O}_{3}$ & - & 0.019 \\
$\mathrm{ZnO}$ & - & 0.102 \\
\hline
\end{tabular}

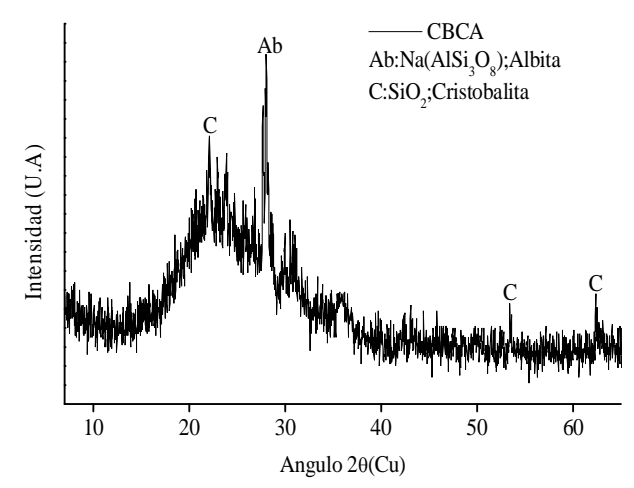

Fig. 1 Patrón de DRX de las CBCA sin reaccionar.

En la Fig. 2 se muestra la fotomicrografía de las CBCA sin reaccionar obtenida por microscopia electrónica de barrido donde su morfología irregular fue apreciable con partículas en forma de bastones, esferas, semiesferas y también su estructura porosa.

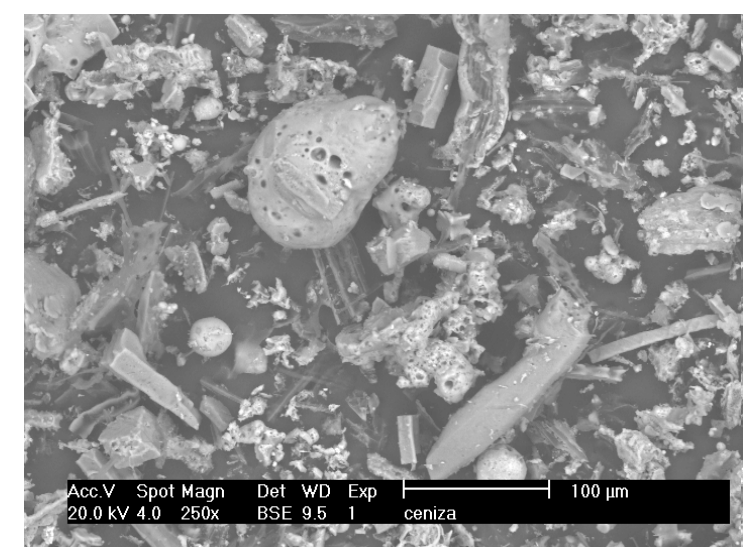

Fig. 2 Fotomicrografía de las CBCA.

En la Fig. 3 se presenta el patrón de difracción del CP obtenido por difracción de rayos X donde se hizo evidente la presencia de fases cristalinas típicas tales como calcita, alita, yeso, belita, aluminato tricálcico o celita y ferrita.

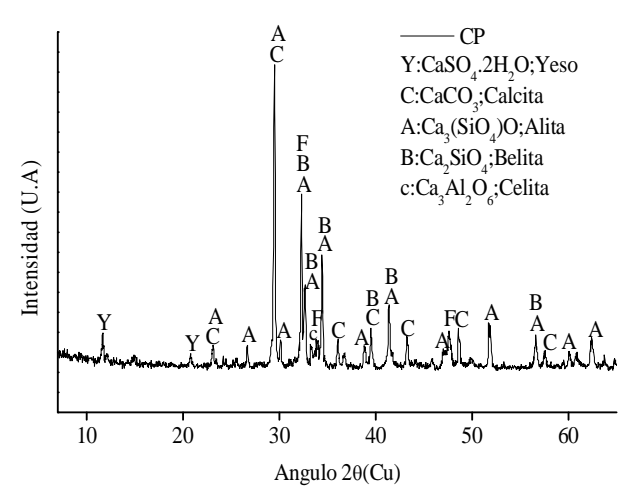

Fig. 3 Patrón de DRX de CP anhidro.

\subsection{Preparación de pastas y probetas cúbicas}

Se fabricaron probetas cúbicas de $2.54 \mathrm{~cm}$ de lado cada una. Las variables estudiadas y sus correspondientes niveles se presentan en la Tabla 2.

Los componentes CP-CBCA y agua fueron mezclados aproximadamente por 5 minutos en 
una mezcladora marca Blazer. La mezcla fue vaciada en las probetas previamente engrasadas, posteriormente, se dejaron fraguar durante 24 horas en una cámara isotérmica a $60{ }^{\circ} \mathrm{C}$. Los especímenes fueron desmoldados colocados en bolsas plásticas, envueltos en paños húmedos y puestos a curar en la cámara isotérmica a $60^{\circ} \mathrm{C}$ hasta la edad correspondiente de prueba (hasta por 28 días).

Tabla 2 Dosificaciones de estudio.

\begin{tabular}{cccc}
\hline Sistema & CP $(\%)$ & CBCA $(\%)$ & $\mathrm{a} / \mathrm{c}$ \\
\hline S1 & 85 & 15 & 0.5 \\
S2 & 55 & 45 & 0.84 \\
Patrón & 100 & 0 & 0.5 \\
\hline
\end{tabular}

a/c: Relación en peso agua/cemento

\subsection{Resistencia mecánica a la compresión de pastas}

Los resultados de resistencia mecánica a la compresión fueron expresados como el promedio de 4 especímenes que fueron ensayados por cada experimento en una prensa hidráulica marca D.A.V.I.S.A a edades de 1, 3, 7, 14 y 28 días de curado.

\subsection{Técnicas experimentales de caracterización}

La caracterización mineralógica fue estudiada por difracción de rayos $\mathrm{X}$ de muestras ensayadas mecánicamente a edad de 7, 14 y 28 días de curado en un difractómetro Phillips PW3040 con un ánodo de cobre.

El estudio se acompañó de análisis termogravimétrico y diferencial (ATG/ATD) simultáneo, de muestras ensayadas a edades de 3 y 28 días en un equipo Perkin- Elmer (modelo Pyris Diamond TG/DTA); las muestras fueron calentadas hasta $1000{ }^{\circ} \mathrm{C}$ a una velocidad de calentamiento de $10{ }^{\circ} \mathrm{C} / \mathrm{min}$ en atmósfera de nitrógeno.

La morfología fue observada utilizando un microscopio electrónico de barrido marca Phillips modelo XL30ESEM (para micrografías $\mathrm{y}$ análisis por EDS) de muestras que no fueron ensayadas mecánicamente a edades de 7, 14 y 28 días de curado.

\section{Resultados y Discusión}

\subsection{Resistencia mecánica a la compresión de pastas}

Los resultados de resistencias a la compresión se presentan en la Tabla 3, los datos entre paréntesis corresponden a la desviación estándar para un promedio de 4 cubos por cada tiempo de prueba; su comportamiento con el tiempo puede visualizarse en la Fig. 4. Se obtuvo que todas las muestras tanto las adicionadas con CBCA y la control incrementan sus resistencias a la compresión con el tiempo de hidratación. Se pudo observar un comportamiento similar en la pasta de referencia y con 15\% CBCA a todas las edades (ver Fig. 4). Las resistencias más altas reportadas fueron de 29.56 y $29.64 \mathrm{MPa}$ para las pastas control y con $15 \%$ CBCA respectivamente, a edad de 28 días. Por el contrario, el comportamiento de la ganancia de resistencia reportadas para la pasta con $45 \%$ CBCA fue muy pobre a todas las edades. En consecuencia a esto, en esta investigación se consideró 15\% CBCA como una proporción óptima de reemplazo parcial al cemento Portland, siempre y cuando el curado se realice a $60{ }^{\circ} \mathrm{C}$ por 28 días.

Los resultados de resistencias mecánicas obtenidos son muy cercanos a los encontrados por (Singh et al., 2000; Ganesan et al., 2007) como un $10 \%$ y $20 \%$ de CBCA como niveles óptimos.

Tabla 3 Resultados de resistencia a la compresión de pastas de CP-CBCA y patrón.

\begin{tabular}{|c|c|c|c|c|c|c|c|c|}
\hline \multicolumn{9}{|c|}{ Resistencia mecánica a la compresión (MPa) } \\
\hline \multirow{2}{*}{ Corrida } & \multirow{2}{*}{$\mathrm{a} / \mathrm{c}$} & \multirow{2}{*}{$\% \mathrm{CP}$} & \multirow{2}{*}{$\% \mathrm{CBCA}$} & \multicolumn{5}{|c|}{ Edad de prueba (días) } \\
\hline & & & & 1 & 3 & 7 & 14 & 28 \\
\hline $\mathrm{S} 1$ & 0.5 & 85 & 15 & $\begin{array}{l}14.6 \\
(1.1)\end{array}$ & $\begin{array}{l}21.0 \\
(0.8)\end{array}$ & $\begin{array}{l}22.4 \\
(2.4)\end{array}$ & $\begin{array}{l}24.9 \\
(1.5)\end{array}$ & $\begin{array}{l}29.6 \\
(1.5)\end{array}$ \\
\hline $\mathrm{S} 2$ & 0.84 & 55 & 45 & $\begin{array}{c}4.2 \\
(0.7)\end{array}$ & $\begin{array}{c}9.3 \\
(0.4)\end{array}$ & $\begin{array}{l}10.4 \\
(0.6)\end{array}$ & $\begin{array}{c}13.0 \\
(1.0)\end{array}$ & $\begin{array}{l}14.5 \\
(0.3)\end{array}$ \\
\hline Patrón & 0.5 & 100 & 0 & $\begin{array}{r}17.5 \\
(1.3) \\
\end{array}$ & $\begin{array}{l}22.5 \\
(2.1) \\
\end{array}$ & $\begin{array}{r}24.6 \\
(3.0) \\
\end{array}$ & $\begin{array}{r}25.0 \\
(1.8) \\
\end{array}$ & $\begin{array}{l}29.5 \\
(2.0) \\
\end{array}$ \\
\hline
\end{tabular}

Dado que la actividad puzolánica se correlaciona con la finura del material (Cordeiro et al., 2009) la baja área superficial y el tamaño de partículas 
de las cenizas produjo poca generación de numerosos sitios de nucleación para la precipitación de productos de hidratación y su alta porosidad provoco un incremento en el requerimiento de agua que conduce a la pérdida de resistencia. Por esto, no se obtuvo un incremento significativo de la resistencia de las pastas sustituidas sobre la resistencia de las pastas de control. Estos factores debieron haber comprometido grandemente la ganancia de resistencias sobre todo en el sistema con $45 \%$ CBCA debido a la mayor cantidad de material adicionado.

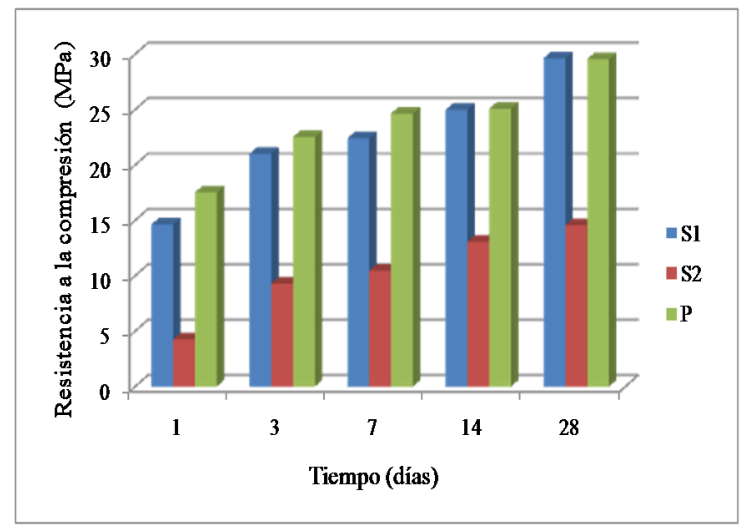

Fig. 4 Desarrollo de la resistencia a la compresión de pastas de CP-CBCA y patrón con el tiempo.

\subsection{Difracción de rayos $X$}

En la Fig. 5 se presentan los difractogramas obtenidos por DRX para las pastas con $15 \%$ CBCA hidratadas a edades de (a) 7, (b) 14 y (c) 28 días. En las muestras en polvos, se encontraron las fases ettringita, portlandita, calcita, kuzelita y $\mathrm{CSH}$ pobremente cristalino como principal producto de hidratación. En los espectros obtenidos, la disminución de la intensidad de los picos correspondientes a la portlandita en $34.1,18.06,47.1,50.82,28.68$ y $54.5^{\circ} 2 \theta$ demuestran el paulatino progreso de la reacción puzolánica entre el $\mathrm{Ca}(\mathrm{OH})_{2}$ del $\mathrm{CP}$ y el $\mathrm{SiO}_{2}$ de la $\mathrm{CBCA}$. Por otra parte esta disminución pudo estar relacionada con la formación de calcita por medio del proceso de carbonatación (Meneses, 2012). La presencia de portlandita aún a los 28 días de curado indicó que la reacción puzolánica no había finalizado.

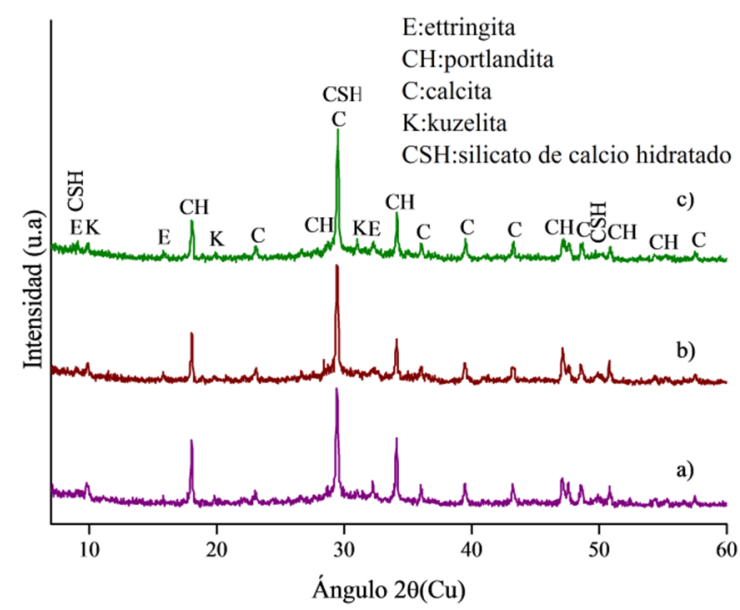

Fig. 5 Patrón de DRX de pastas con 15\% CBCA a) 7 días; b) 14 días; c) 28 días de curado.

Resulta difícil la identificación de un solo tipo de CSH porque su composición varía con el tiempo de hidratación (Cong y Kirkpatrick, 1996). Las fases de gel CSH encontradas a todas las edades corresponden a los compuestos tobermorita y nekoita cuyas reflexiones principales se encuentran en $29.50,49.92^{\circ} 2 \theta$ y $9.72,29.50,49.70^{\circ} 2 \theta$, respectivamente. Estos resultados concuerdan con la literatura donde el gel CSH es caracterizado por tres picos localizados en $29.34,32.04$ y $50.06^{\circ} 2 \theta$ en muestras de cemento Portland (Lecomte et al., 2006).

En la Fig. 6 se muestran los difractogramas obtenidos por DRX para las pastas con $45 \%$ CBCA hidratadas a edades de (a) 7, (b) 14 y (c) 28 días. En las muestras fueron encontradas las fases ettringita, portlandita, cuarzo y $\mathrm{CSH}$ pobremente cristalino como principal producto de hidratación correspondiente al compuesto reinhardbraunsita cuyas reflexiones principales se ubican en $29.41,32.25$ y $47.41^{\circ} 2 \theta$.

De igual forma que en el sistema con $15 \%$ CBCA disminuyó la intensidad de los picos de portlandita solamente entre los 7 y 14 días esto indicó que esta se consumió, ya sea, en el proceso de carbonatación o en la reacción puzolánica. La presencia de portlandita fue nula a los 28 días de curado, lo que demostró que probablemente finalizó la reacción puzolánica 
por poco suministro de portlandita, quedando un exceso de CBCA sin reaccionar.

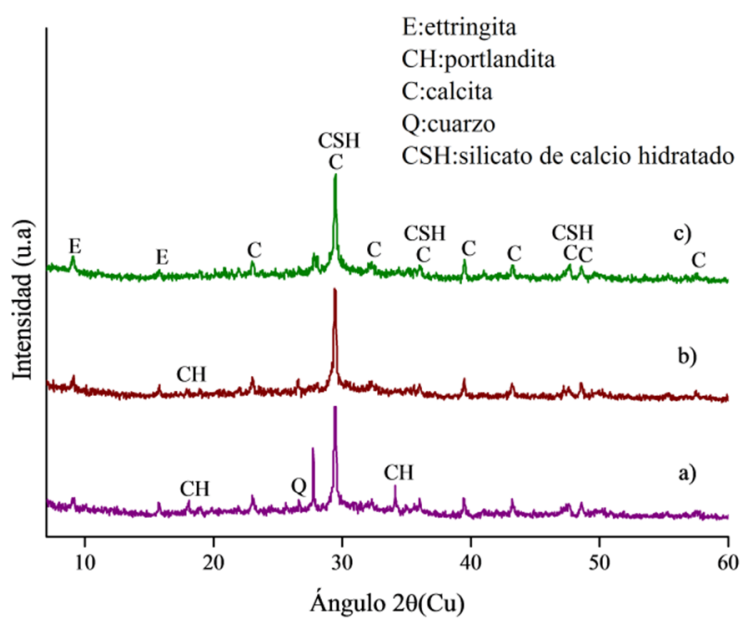

Fig. 6 Patrón de DRX de pastas con $45 \%$ CBCA a) 7 días; b) 14 días; c) 28 días de curado.

\subsection{Análisis Térmico}

A través del análisis termogravimétrico (ATG) es posible cuantificar y evaluar las pérdidas de peso que sufre la muestra con la temperatura. Con el análisis térmico diferencial (ATD) se logra identificar los rangos de temperatura en los cuales ocurren las transformaciones de las fases presentes. En la Fig. 7 se presentan los termogramas de las pastas con (a) 15\% CBCA y (b) $45 \%$ CBCA.

Se determinó a simple vista en las curvas de ATG las pendientes pertenecientes a la descomposición de la portlandita y calcita (Meneses, 2012), que fueron localizadas para ambos sistemas entre los $400-450{ }^{\circ} \mathrm{C}$ y $600-800$ ${ }^{\circ} \mathrm{C}$, las cuales se encontró que disminuyen con el incremento en el porcentaje de sustitución de $\mathrm{CP}$ por CBCA y la edad de curado, esto es apreciable sobre todo para la portlandita donde su presencia fue nula en la pasta con $45 \%$ CBCA, coincidiendo con la técnica de DRX. Puede notarse además, el pico endotérmico de la calcita, el cual se mostró más pequeño, puede asumirse que el proceso de descomposición de esta fase absorbe menos energía.

La descomposición de la portlandita y calcita es atribuible a la perdida de agua y dióxido de carbono, respectivamente. Según el análisis por ATD dicha descomposición ocurrió mediante procesos endotérmicos. También fueron localizados picos entre los $50-150{ }^{\circ} \mathrm{C}$ y los 200 $400{ }^{\circ} \mathrm{C}$ posiblemente pertenecientes a la pérdida de peso del gel CSH (Flores, 2009) y Ettringita (Escalante-García, 2002).
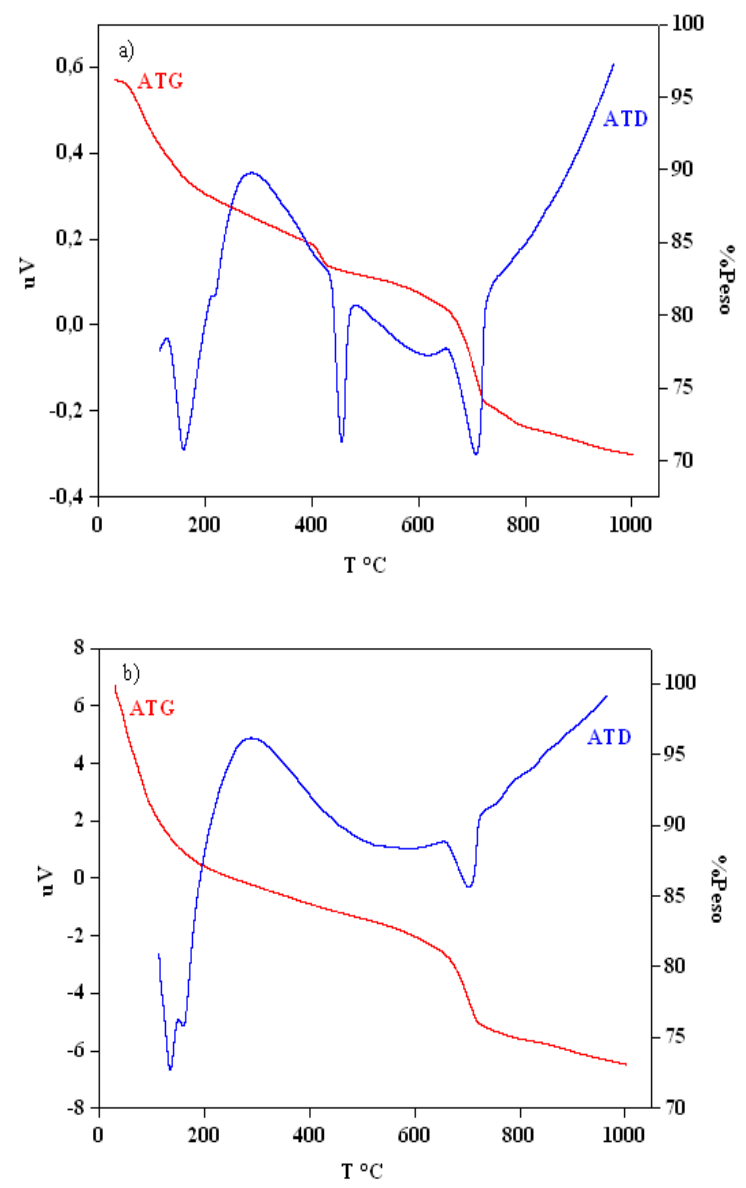

Fig. 7 Termogramas de los sistemas a) $15 \%$ CBCA y b) $45 \%$ CBCA curados a 28 días.

\subsection{Microscopía electrónica de barrido}

En la Fig. 8 se presentan las microestructuras de muestras que fueron analizadas a 28 días de curado. Las imágenes fueron obtenidas por electrones retrodispersados distinguiendo las fases, como lo indica la literatura, según el orden de brillantez proporcional al número atómico de la zona irradiada (Escalante-García, 2002).

En la Fig. 8A se muestra la microestructura de la pasta con $15 \%$ CBCA (29.64 MPa a 28 días) y 
en la Fig. 8 B se muestra la microestructura de la pasta con $45 \%$ CBCA (14.58MPa a 28 días). La menor RM para la pasta con $45 \%$ CBCA es atribuible a la presencia de una gran cantidad de cenizas sin reaccionar que en comparación con la microestructura de la pasta con $15 \%$ CBCA es menor; a esto se suma el poco suministro de $\mathrm{Ca}(\mathrm{OH})_{2}$ por lo que la reacción puzolánica pudo ocurrir muy rápidamente provocando que los productos de hidratación no alcanzaran a difundir y depositarse en forma homogénea (Espinoza-Pérez y Escalante-García, 2011).

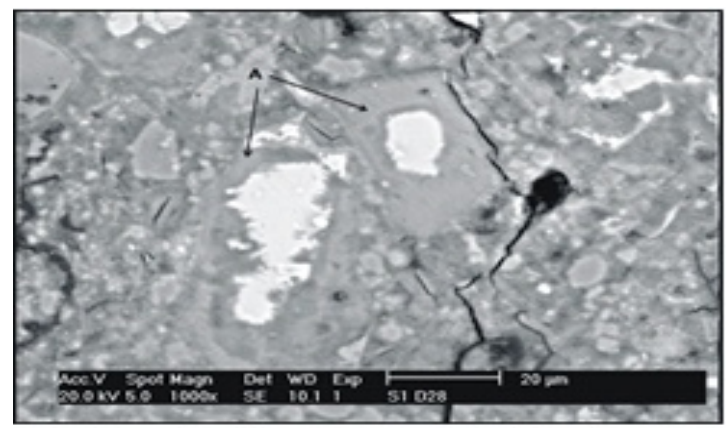

A

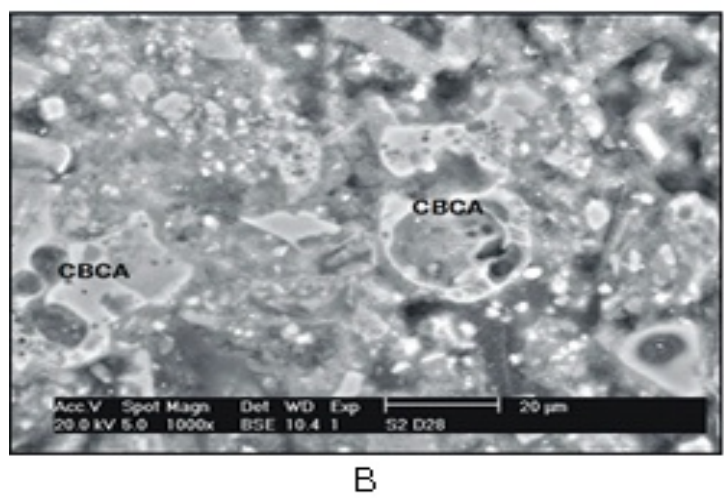

Fig. 8 Microestructura de pastas A) $15 \%$ CBCA y B) $45 \%$ CBCA.

En la microestructura de la pasta con $15 \%$ CBCA se observó el mecanismo de reacción en estado sólido debido a la presencia de anillos de reacción alrededor de los granos de cemento parcialmente hidratados que fueron claramente apreciables y una matriz más densa, y compacta. Además, es de esperarse que aún después de los 28 días puedan darse ganancias de resistencia mecánica debido a que todavía faltan granos que no han reaccionado completamente con la sílice reactiva de las cenizas.

\section{Conclusiones}

Las CBCA contienen $62.33 \%$ de $\mathrm{SiO}_{2}$. La sílice reactiva presente se demuestra en el halo amorfo obtenido en la prueba por DRX.

Las CBCA utilizadas actúan como una puzolana que puede sustituir al $\mathrm{CP}$ hasta en un $15 \%$ en peso como reemplazo óptimo, según las condiciones establecidas en esta investigación. Mayores porcentajes de sustitución producen detrimentos de las propiedades mecánicas.

Las observaciones en la microscopía electrónica de barrido (MEB) a la microestructura de las pastas CP-CBCA demuestran la formación de productos de hidratación en presencia del mecanismo de reacción en estado sólido, debido, a la formación de anillos de reacción alrededor de los granos de CP parcialmente reaccionados. Además se obtuvo una microestructura del sistema con $45 \%$ CBCA menos densa y compacta en acuerdo a los resultados de resistencia a la compresión.

Las curvas obtenidas por ATG/ATD confirman la formación de los productos de hidratación. También se demostró que en las pastas con mayor porcentaje de CBCA se consume más rápidamente el $\mathrm{Ca}(\mathrm{OH})_{2}$, que pudo deberse al exceso de cenizas y poca disponibilidad del $\mathrm{Ca}(\mathrm{OH})_{2}$ originado en la hidratación del CP.

\section{Agradecimientos}

Los autores agradecen al Proyecto CB2012/182424 de CONACYT (México) que financió la investigación. Además, agradecen a la corporación Pantaleón, Ingenio Monte Rosa por la colaboración brindada. Así mismo se agradece a la Facultad de Ingeniería Química (FIQ) y la Facultad de Tecnología de la Construcción (FTC) de la UNI por el apoyo.

\section{Referencias}

Alujas, A., Fernández, R., Martinera, J.F., Quintana, R. (2010). Empleo de arcillas caoliníticas de bajo grado activadas térmicamente como una alternativa para 
reemplazo parcial de cemento Pórtland. CENIC. Ciencias Químicas, 41, 1-10.

Carmona, P. (2011). Evaluación mecánica de pastas de cemento modificadas con escoria de horno de arco eléctrico y ceniza de bagazo de caña de azúcar. Tesis de grado. Facultad de Ingeniería Civil, Universidad Veracruzana.

Cong, X., Kirkpatrick, R.J. (1996). 29 Si MAS NMR study of the structure of calcium silicate hydrate. Advn Cem Bas Mat, 3, 144-156.

Cordeiro, G.C., Toledo Filho, R.D., Fairbairn, E.M.R. (2009). Effect of calcination temperature on the pozzolanic activity of sugar cane bagasse ash. Constrution and building materials, 23, 3303.

Cordeiro, C.G., Toledo Filho, R.D., Tavares, L.M., Fairbairn, E.M.R. (2009). Ultrafine grinding of sugar cane bagasse ash for application as pozzolanic admixture in concrete. Cement and Concrete Research, 39, 110-115.

Escalante, J.I. (2002). Materiales alternativos al cemento Pórtland. Avance y Perspectiva, 21, 7988 .

Escalante-García, J.I. (2002). Curso de Cementos, Maestría en Ciencias en Ingeniería Cerámica, Cinvestav, México.

Espinoza-Pérez, L.J., Escalante-García, J.I. (2011). Morteros a base de vidrio de desecho/escoria de alto horno; activación mecanoquímica del vidrio en soluciones alcalinas. Nexo, 24(2), 92-103.

Flores, I. (2009). Obtención de nanopartículas de $\mathrm{SiO} 2$ y su efecto en las propiedades microestructurales, mecánicas y reológicas en materiales cementantes. Tesis Doctoral. Facultad de Ingeniería Civil, Universidad Autónoma de Nuevo León.
Ganesan, K., Rajagopal, K., Thangavel, K. (2007). Evaluation of bagasse ash as suplementary cementitious material. Cement \& Concrete Composites, 29,515-524.

Lecomte, I., Henrist, M., Li'egeois F., Maseri, F., Rulmont, A., Cloots, R. (2006). (Micro)structural comparison between geopolymers, alkali-activated slag cement and Portland cement. Journal of the European Ceramic Societ.

Martinera, J.F., Middendorf, B., Gehrke, M., Budelmann, H. (1998). Use of wastes of the sugar industry as pozzolana in lime-pozzolana binders: study of the reaction. Cement and Concrete Research, 28(11), 1525-1536.

Meneses, N. (2012). Evaluación de la reacción de carbonatación en los estudios de las fases de conglomerados con cemento Pórtland. Tesis de grado. Universidad Politécnica de Catalunya.

Morales, E.V., Villar-Cociña, E., Frias, M., Santos, S.F., Savastano Jr, H. (2009). Effects of calcining conditions on the microestructure of sugar cane waste ashes (SCWA): influence in the pozzolanic activation. Cement \& Concrete Composites, 31, 22-28.

Payá, J. (2012). La "transmutación" sostenible de los residuos para nuevas materias primas en el ámbito del concreto. DYNA, 79(175), 38-47.

Perdomo, J.R. (2009). Informe laboral, social y ambiental. Corporación Pantaleon.

Quintana, E. (2005). Relación entre las propiedades geotécnicas y los componentes puzolánicos de los sedimentos pampeanos. Tesis Doctoral. Facultad de Ciencias, Universidad Nacional de Córdoba.

Singh, N.B., Singh, V.D., Rai, S. (2000). Hydratation of bagasse ash-blended Portland cement. Cement and Concrete Research, 30, $1485-1488$. 